Interfaces and Free Boundaries 15 (2013), 181-202

DOI $10.4171 / \mathrm{IFB} / 300$

\title{
Anomalous diffusion models in the presence of a moving interface
}

\author{
CHRISTOPHER A. GRUBER \\ Department of Engineering Sciences and Applied Mathematics, \\ Northwestern University, 62008 Evanston, USA \\ E-mail: christophergruber2012@u.northwestern.edu \\ CHRISTOPHER J. VOGL \\ Department of Engineering Sciences and Applied Mathematics, \\ Northwestern University, 62008 Evanston, USA \\ E-mail:cvogl@u.northwestern.edu \\ MiChAEL J. MiKsis \\ Department of Engineering Sciences and Applied Mathematics, \\ Northwestern University, 62008 Evanston, USA \\ E-mail:miksis@northwestern.edu \\ STEPHEN H. DAVIS \\ Department of Engineering Sciences and Applied Mathematics, \\ Northwestern University, 62008 Evanston, USA \\ E-mail:sdavis@northwestern.edu
}

[Received 16 April 2012 and in revised form 28 February 2013]

\begin{abstract}
Many systems exhibit subdiffusive transport in which a diffusing particle's mean-squared displacement has a time dependence that is slower than linear. Here, we study a model of subdiffusion, scaled Brownian motion (SBM), in the context of two-phase moving-boundary problems. In certain cases, the problems admit similarity solutions, though, in general, numerical approaches are required. Turn-around of the moving interface is observed when one domain exhibits subdiffusive transport and the other classical diffusive transport. In each case, the SBM dynamics is compared with another model of subdiffusion, fractional anomalous diffusion (FAD). In the limit that the subdiffusive region is nearly-classical in nature we explore the notion of using SBM as an approximation to FAD. One advantage of this approach is that computations involving SBM are less intensive than those for the corresponding FAD models.
\end{abstract}

2010 Mathematics Subject Classification: Primary 35R37; Secondary 49K20.

Keywords: Anomalous diffusion; moving-boundary problem; glass transition; biopreservation.

\section{Introduction}

Brownian motion, as described by the diffusion equation, captures transport dynamics in a wide variety of materials. Its characteristic feature is a mean-squared displacement (MSD) having a linear time dependence. This classical (Brownian) diffusion process describes transport in many situations, though a number of materials exhibiting nonlinear MSDs has been observed experimentally. These so-called anomalous diffusion processes have the property that their MSD is nonlinear in time. Due to the fact that these anomalous diffusion processes have received substantially less attention than 
their classical counterparts, their dynamics is not as well understood as is Brownian motion. Here, we study a class of moving boundary problems in which the dynamics entails a slower-than-linear MSD (subdiffusion).

There are a number of mathematical models that account for diffusion processes with sublinear MSDs. In this paper, scaled Brownian motion (SBM) models are considered; this process is related to classical diffusion by rescaling time. This model is compared to fractional anomalous diffusion (FAD), a model which is derived using a continuous-time random walk in which diffusing particles have waiting-times distributed according to a power-law distribution (by contrast, particles have exponentially-distributed waiting times in the classical treatment). Although both of these anomalous diffusion processes have the same MSD time scaling, the FAD model entails a convolution integral in time that links a system's dynamics to its history, whereas the SBM model has no such memory property. As a result, solving the governing equations of a diffusion process obeying SBM dynamics is simpler and less computationally expensive than for the corresponding FAD system. The SBM model is derived "from the top down" in the sense that the MSD corresponding to classical diffusion can be altered by rescaling time so that the MSD has nonlinear time dependence. The similarity between the evolution predicted by this model and FAD justifies a study to distinguish them. Limiting cases where SBM and FAD yield similar results will be emphasized in order to better understand the differences between the two models. The conclusion is that using SBM in place of FAD allows one to employ a less complex model of a subdiffusive phenomenon while retaining the salient features of the dynamics. In particular, for higher dimensions this replacement takes an extremely complicated and computationally intensive investigation into a more classical setting where established methods can be used.

In this paper, we consider two-phase moving-boundary problems in which one or both media exhibit subdiffusion. In a moving-boundary problem, a mobile interface separates two distinguishable media; the propagation of this interface is driven by the diffusion of heat or mass through the domain (the melting of polar ice in the Arctic is an example of such a system). The location of this moving interface is an unknown quantity of the problem.

Our investigation is motivated by studies of biopreservation by way of desiccation and vitrification (conversion to a glassy phase), a technique known as lyopreservation [27]. A number of anhydrobiotic organisms (yeasts, nematodes, etc.), when encountering harsh environmental conditions, undergo anhydrobiotic preservation and emerge as viable organisms when more favorable conditions arise [8]. Some organisms, such as the seeds of certain plants, have been known to survive decades in the preserved state [8]. The vitrification of biological samples is induced through the injection of special sugars (e.g. trehalose $[4,5,15])$ into the cells and the loss of water through the cell membranes. These sugars have been shown to have an essential role in dramatically slowing intracellular transport processes [1], stabilizing cell membranes [8, 9], and preventing cell membrane phase changes $[7-9,11]$. The virtues of anhydrobiosis as a preservation technique (as compared to cryogenesis, for example) are that the process occurs at ambient temperature and that the preserved tissues are much lighter than the original material, thus reducing storage and transport costs [13].

The vitrification transition is characterized by a continuous but dramatic increase in the liquid's viscosity [3], giving glassy substances solid-like mechanical properties despite the lack of a regular crystalline structure [10]. Glassy phases are a subject of considerable current interest. One recentlydeveloped model of glasses treats these materials as aperiodic crystals [17]. From the perspective of mathematical modeling, the conversion of a liquid solution to a glassy state is interesting because 
diffusion in this glassy state has been shown to exhibit subdiffusive dynamics over short time scales, hence providing motivation for this work $[6,25]$. Although subdiffusion is a subject of much attention in the literature (e.g., [21, 22]), the models considered in the present paper are novel due to the presence of moving boundaries.

Subdiffusion is characterized by a mean-squared displacement that is sublinear in time, i.e., $\left\langle x^{2}(t)\right\rangle \sim t^{\alpha}$, where $0<\alpha<1$. A number of mathematical models demonstrate this scaling of the second moment, including fractional Brownian motion [20], generalized Langevin equations [18], scaled Brownian motion [16], and FAD [22]. Metzler and Klafter provide an overview of the various subdiffusive processes in their review article [22]. For the case of glassy materials, Weeks and Weitz [25] contend that glasses exhibit FAD dynamics; the memory property of the FAD operator is accounted for by the experimental observation that particles tend to crowd into clusters (the socalled "caging" phenomenon). It should be noted that superlinear $(\alpha>1)$ MSD behavior has also been observed experimentally in so-called superdiffusive materials. These models are not considered here; a discussion can be found in [22].

In the present paper, we apply analytical and numerical methods to the problem of subdiffusion in the presence of moving interfaces. First, we consider situations in which subdiffusion occurs on both sides of an interface. For the special case where the order of the derivative is the same on both sides, a similarity solution is found. Next, guided by the desiccation experiments noted above, we consider situations in which subdiffusion occurs on one side of an interface and classical diffusion on the other. This situation is then generalized by considering two subdiffusive regions with dissimilar anomalous diffusion parameters. Finally, SBM is investigated as an approximation to FAD by considering a problem with one classical diffusion region and one FAD region in which the order of fractional derivative is near the classical diffusion limit.

The focus of this paper is scaled Brownian motion and the relationship of the model to FAD. A more extensive numerical study of the dynamics of interfaces governed by FAD can be found in [24]. It will be shown that SBM can be recovered, to leading order, from FAD dynamics in the limiting case where the order of the fractional derivative approaches the classical diffusion case (i.e., $\alpha \rightarrow 1)$. It will also be shown that the qualitative behavior of the dynamics of an interface predicted by the two models is very similar, and that a proper redefinition of the diffusion coefficient can minimize this difference. Although the error in replacing the FAD operator with the SBM operator is not uniform in time, the results are encouraging and suggest additional work to improve the approximation.

\section{Anomalous diffusion}

The term subdiffusion describes any diffusive transport process for which the mean-squared displacement (MSD) of a particle depends on time in accordance with a sublinear power-law, i.e.,

$$
\left\langle x^{2}(t)\right\rangle \sim t^{\alpha},
$$

where $0<\alpha<1$. One model exhibiting this MSD pattern is fractional anomalous diffusion (FAD). The dynamics of this model is described by the fractional PDE

$$
C_{t}(x, t)=d_{f} \mathbf{D}_{t}^{1-\alpha} C_{x x}(x, t),
$$

where ${ }_{a} \mathbf{D}_{t}^{1-\alpha}$ is the Riemann-Liouville fractional derivative operator. This operator can be thought of as the composition of a fractional integral ${ }_{a} \mathbf{D}_{t}^{-\alpha}$ and a conventional derivative. The subscripts $a$ 
and $t$ refer to the lower and upper limits of integration, respectively, and the superscript is the order of the fractional operator. In the notation adopted here, the Riemann-Liouville operator takes the form

$$
{ }_{a} \mathbf{D}_{t}^{1-\alpha} f(t)=\frac{1}{\Gamma(\alpha)} \frac{\partial}{\partial t} \int_{a}^{t}\left(t-t^{\prime}\right)^{-1+\alpha} f\left(t^{\prime}\right) d t^{\prime},
$$

valid for $0<\alpha \leqslant 1$, where $\Gamma(x)$ is the gamma function. The FAD equation can be rigorously derived by way of a continuous-time random walk in which the diffusing particles have a waitingtime distribution with long-time asymptotic behavior obeying the power-law $t^{-2+\alpha}$ (see, for example, [21]). When the lower terminus is $a=0$, the FAD equation has the propagator [21]

$$
C(x, t)=\frac{1}{\sqrt{4 \pi d_{f} t^{\alpha}}} H_{1,2}^{2,0}\left[\frac{x^{2}}{4 d_{f} t^{\alpha}} \mid \begin{array}{l}
(1-\alpha / 2, \alpha) \\
(0,1),(1 / 2,1)
\end{array}\right]
$$

and MSD

$$
\left\langle x^{2}(t)\right\rangle=\frac{2 d_{f}}{\Gamma(1+\alpha)} t^{\alpha} .
$$

In (2.4), the function $H$ is the Fox function, a very general special function [21]. The Fox function is defined in terms of a series; the propagator (2.4) is equivalent to the expression

$$
C(x, t)=\frac{1}{\sqrt{4 d_{f} t^{\alpha}}} \sum_{n=0}^{\infty}(-1)^{n} \frac{1}{n ! \Gamma(1-\alpha(n+1) / 2)}\left(\frac{x^{2}}{d_{f} t^{\alpha}}\right)^{n / 2} .
$$

In the limit that $\alpha \rightarrow 1,(2.4)$ recovers the classical propagator

$$
C(x, t)=\frac{1}{\sqrt{4 \pi d_{f} t}} e^{-\frac{\alpha x^{2}}{4 d_{f} t}}
$$

by virtue of the Taylor series for the exponential function,

$$
e^{x}=\sum_{n=0}^{\infty} \frac{1}{\Gamma(n+1)} x^{n}
$$

Another model of subdiffusion is scaled Brownian motion (SBM). The governing equation for this process is derived by rescaling the time variable in the classical diffusion model as $t^{*}=\frac{1}{\alpha} t^{\alpha}$ so that the diffusion equation is

$$
C_{t^{*}}\left(x, t^{*}\right)=d_{s} C_{x x}\left(x, t^{*}\right) .
$$

In the original time variable, this transformation yields the equation [16]

$$
C_{t}(x, t)=d_{s} t^{-1+\alpha} C_{x x}(x, t) .
$$

By analogy to the FAD equation, the exponent $-1+\alpha$ will be referred to as the order of the SBM process. Equation (2.10) has the propagator

$$
C(x, t)=\frac{\sqrt{\alpha}}{\sqrt{4 \pi d_{s} t^{\alpha}}} e^{-\frac{\alpha x^{2}}{4 d_{s} t^{\alpha}}}
$$


and MSD

$$
\left\langle x^{2}(t)\right\rangle=\frac{2 d_{s}}{\alpha} t^{\alpha} .
$$

Note that the MSD of the two models can be equated by rescaling the diffusivity as

$$
d_{s}=\frac{1}{\Gamma(\alpha)} d_{f}
$$

Even though the models can be made to have the same MSD, the dynamics of systems governed by these equations can be quite different. In particular, the FAD equation is an integro-differential equation, whereas the SBM dynamics obeys the classical diffusion PDE with a time-dependent diffusivity. As a result of the temporal convolution integral in FAD (equation (2.2)), the current state of the system depends on all the previous states, weighted by a power-law kernel. The FAD model is, hence, subdiffusive. SBM (equation (2.10)) has no such memory term; its anomalous behavior arises from the explicit time-dependent coefficient in the differential equation [16]. Despite this important qualitative difference between the models, both converge quantitatively to classical diffusion dynamics in the limit that $\alpha \rightarrow 1$. Taking $\alpha=1-\epsilon$ with $0<\epsilon \ll 1$ and expanding for small $\epsilon$, both propagators can be shown to equal

$$
C(x, t ; \epsilon)=\frac{1}{\sqrt{4 \pi d_{i} t^{1-\epsilon}}} e^{-\frac{x^{2}}{4 d_{i} t^{1-\epsilon}}}+\mathrm{O}(\epsilon),
$$

where $d_{i}=d_{f}$ for FAD and $d_{i}=d_{s}$ for SBM.

One important question about the propagators (2.4) and (2.11) concerns the time dependence of the solution in the vicinity of the singularity (here $x=0$ ) for small time. For FAD, the propagator (2.4) can be expressed as the series [21]

$$
C(x, t)=\frac{1}{\sqrt{4 d_{f} t^{\alpha}}} \sum_{n=0}^{\infty}(-1)^{n} \frac{1}{n ! \Gamma(1-\alpha(n+1) / 2)}\left(\frac{x^{2}}{d_{f} t^{\alpha}}\right)^{n / 2} .
$$

In the limit $x \rightarrow 0$, the modes $n>0$ vanish, yielding the small- $x$ result

$$
\lim _{x \rightarrow 0} C(x, t)=\frac{1}{2 \sqrt{d_{f}} \Gamma(1-\alpha / 2)} t^{-\alpha / 2}=k_{f} t^{-\alpha / 2} .
$$

For SBM, the propagator (2.11) has the small- $x$ behavior

$$
\lim _{x \rightarrow 0} C(x, t)=\frac{\sqrt{\alpha}}{2 \sqrt{\pi d_{s}}} t^{-\alpha / 2}=k_{s} t^{-\alpha / 2} .
$$

The propagators have small- $x$ behavior proportional to $t^{-\alpha / 2}$ with coefficients dependent upon the diffusivity and $\alpha$. Both propagators approach the classical result

$$
\lim _{x \rightarrow 0} C(x, t)=\frac{1}{2 \sqrt{\pi d_{s}}} t^{-1 / 2}
$$

as $\alpha \rightarrow 1$ in a smooth (non-singular) fashion. 
An additional result in the small- $\epsilon$ limit is that the Riemann-Liouville operator can be expanded for small $\epsilon$ to yield the scaled Brownian motion operator plus a correction. Consider the RiemannLiouville derivative with order $\epsilon$, where $0<\epsilon \ll 1$, acting on a generic function of time $f(t)$ :

$$
{ }_{\mathrm{a}} \mathbf{D}_{t}^{\epsilon} f(t)=\frac{1}{\Gamma(1-\epsilon)} \frac{d}{d t} \int_{a}^{t}\left(t-t^{\prime}\right)^{-\epsilon} f\left(t^{\prime}\right) d t^{\prime} .
$$

Using integration by parts, evaluating the derivative, and rearranging, the operator can be shown to be

$$
{ }_{\mathrm{a}} \mathbf{D}_{t}^{\epsilon} f(t)=\frac{1}{\Gamma(1-\epsilon)}(t-a)^{-\epsilon} f(t)+I,
$$

where

$$
I=\frac{1}{\Gamma(1-\epsilon)} \int_{a}^{t}\left(\left(t-t^{\prime}\right)^{-\epsilon}-(t-a)^{-\epsilon}\right) \frac{d}{d t^{\prime}} f\left(t^{\prime}\right) d t^{\prime} .
$$

Making use of the Cauchy-Schwarz inequality, the expression $I$ obeys the inequality

$$
|I| \leqslant \epsilon M(t-a),
$$

where $M$ is the maximal value of $\frac{d}{d t^{\prime}} f\left(t^{\prime}\right)$ on the interval $t^{\prime} \in[a, t]$.

Under certain conditions, $I$ can be neglected; this simplification converts the expression (2.20) into an approximation to the Riemann-Liouville operator (2.2). The inequality (2.22) is o(1) provided that the quantity $M(t-a)$ is $\mathrm{O}(1)$. This condition is satisfied if $M$ is bounded and the time variable obeys $t-a \ll \frac{1}{M}$. In practice, $C_{t x x}(x, t)$ is bounded and its value is small enough that the act of approximating the Riemann-Liouville operator (2.2) by the first term of (2.20) is valid up to $(t-a)=\mathrm{o}\left(\frac{1}{\epsilon}\right)$. This result implies that SBM is a good approximation for FAD in the nearly-classical limit $\alpha \rightarrow 1$ for small to moderate times.

Motivated by the leading-order expansion (2.20) and the fact that SBM and FAD have meansquared displacements with the same time dependence, SBM will be studied as a model of subdiffusion. The primary incentive for studying SBM is its lack of the memory property associated with FAD. Due to this memory, numerical solutions of subdiffusion problems using FAD tend to be very computationally intensive. Using SBM as a model for subdiffusion could potentially yield qualitatively similar solutions with less use of numerical resources.

Here, several free-boundary problems will be investigated. First, the special case is solved where the two regions bounding the moving interface obey SBM dynamics of the same order but with different diffusivities. Because the order is the same on both sides of the interface, a similarity variable can be identified and an exact analytical solution found. This solution will be compared to the numerical results from [24] for the identical problem with FAD in place of SBM. Next, the case of one SBM and one classical diffusion region will be considered; solutions will be obtained by appealing to finite difference methods. The solutions for this situation reveal interesting dynamics concerning the dynamics of a moving front. Next, we consider the most general case of two SBM regions with dissimilar order. This problem also requires the use of numerical methods and is phenomenologically similar to the case of one anomalous and one classical region. Finally, we consider the case of one classical diffusion region and one FAD region in which the order is near the classical diffusion limit. By making use of approximation (2.20), a leading-order asymptotic solution is obtained in a semi-infinite geometry. The solutions yield insight into the use of the SBM model as a stand-in for FAD. 


\section{Formulation of the problem}

Although our aim is to study several one-dimensional moving-boundary problems governed by SBM (equation (2.10)), it is instructive to first formulate the mathematical problem associated with FAD (equation (2.2)).

Consider the 1D domain $x \in(-\infty, \infty)$ with a moving interface at $x=h(t)$ which separates the anomalous region with diffusivity $d_{1}$ and order $1-\alpha$ from a region with diffusivity $d_{2}$ and order $1-\beta$, where $0<\alpha, \beta \leqslant 1$. Suppose that, initially, $h(0)=0$ and, without loss of generality, assume that $\dot{h}(t)>0$ (i.e., the interface is initially moving to the right). Name the three portions of the domain region I $(-\infty<x<0)$, region II $(0<x<h(t))$, and region III $(h(t)<x<\infty)$; see Figure 1 for an illustration. Note that region II is formed as the interface propagates and has material with properties different from those in region I. Denote by $s(x)$ the time that the front passes the spatial location $x$. With a rightward-moving front, we have that $s(x) \equiv h^{-1}(x)$ and the material in the region II has age $t-s(x)$. This distinction between old and new material properties necessitates a fixed boundary at $x=0$. The boundary conditions imposed at this point are discussed below. The more complicated situation of an interface that reverses its direction of propagation will arise in Section 5.

The lower limit of integration in (2.2) depends on spatial location. For example, for a rightwardmoving interface, regions I and III have lower limit $a=0$ because the material existed at the initial time. For the material in region II, the age is less than the current time; this history should trace back to the time at which the front swept through the point, i.e., $s(x)$. Note that alternative treatments of the history exist in the literature. Some authors (e.g., [12]) take the history at each point $x$ back to the initial time by allowing the material to "remember" its history, irrespective of material changes. The point of view taken here is that a material's memory begins anew when a transition occurs. In other words, the amount of material at a spatial point is "locked in" when a transition front sweeps through that point.

The model for anomalous diffusion used here is based on the FAD model introduced in [24]. There, the FAD model of this moving-boundary problem is derived in the Appendix of [24] using a continuous-time random walk approach and by assuming an exponentially-decaying jump length probability density function (pdf) and a Poisson-like waiting time pdf in the anomalous region. The resulting governing equation in region II is

$$
C_{t}(x, t)=d_{f(x)} \mathbf{D}_{t}^{1-\alpha} C_{x x}(x, t),
$$

where $s(x)$ is replaced by 0 in the regions $x \in(-\infty, 0)$ and $x \in(h(t), \infty)$. Note that the governing equation is not in divergence form; it is possible to rephrase the equation in this form (see the

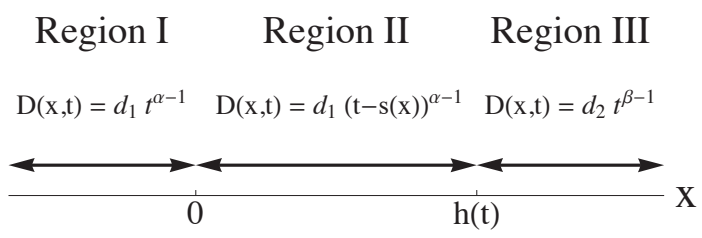

FIG. 1. The three regions of the scaled Brownian motion problem. The region $-\infty<x<h(t)$ is divided into two due to the fact that $s(x)$ is piecewise-continuous at $x=0$. At this point, there is an intersection between the glassy material from the initial data $(x<0)$ and glassy material formed as the drying front propagates $(0<x<h(t))$. Since $s(x)$ obeys $s(x) \rightarrow 0$ in the limit that $x \rightarrow 0^{+}$, the function is piecewise continuous at the point $x=0$. 
discussion below), but it is convenient for our purposes to leave the expression as-is. Using the expansion (2.20), a leading-order formulation for the SBM model can be obtained for $a=s(x)$ and in the limit of $\alpha$ near 1 . Because our objective is to investigate the appropriateness of SBM as a model of subdiffusion as compared to FAD, we relax the assumption that $\epsilon$ be small in this expansion. This approach yields a model for SBM with $0<\alpha \leqslant 1$ (see below) that recovers the classical behavior in the limit of $\alpha \rightarrow 1$.

As noted, the main interest here is anomalous diffusion governed by SBM, equation (2.10). The formulation of an initial boundary-value problem with a moving interface for SBM parallels the FAD case. In particular, the regions in which $s(x)$ is zero and nonzero are identical to the problem above. The PDE for SBM is

$$
C_{t}(x, t)=D(x, t) C_{x x}(x, t) .
$$

where

$$
D(x, t)= \begin{cases}d_{1} t^{-1+\alpha}, & -\infty<x<0, \\ d_{1}(t-s(x))^{-1+\alpha}, & 0<x<h(t), \\ d_{2} t^{-1+\beta}, & h(t)<x<\infty .\end{cases}
$$

The division of two initial regions into three for $t>0$ is illustrated in Figure 1.

Boundary conditions must be specified at the point $x=0$ and at the moving interface $x=h(t)$. The point $x=0$ has the same material on either side, so continuity of the concentration $C(x, t)$ and the flux is imposed. Continuity of the concentration, continuity of the flux, and critical value of the concentration $C(x, t)=c^{*}$ are imposed at the interface $x=h(t)$. For the case of anomalous diffusion on the left and classical diffusion on the right, the boundary condition $C(h(t), t)=c^{*}$ can be interpreted as the solution-to-glass transition criterion (i.e., the critical concentration associated with the glass transition). In the more general case of two dissimilar anomalous regions, $\alpha \neq \beta$ and $\alpha, \beta<1$, the condition simply defines the moving interface between the phases.

Unless otherwise noted, when considering the problem on the real line, we assume the initial data

$$
C(x, 0)=c^{0} H(x),
$$

where $H(x)$ is the Heaviside function, and the far-field boundary data

$$
C(-\infty, t)=0
$$

and

$$
C(\infty, t)=c^{0} .
$$

At the moving interface $x=h(t)$,

$$
C(h(t), t)=c^{*} .
$$

The quantities $c^{0}$ and $c^{*}$ are constants.

The final condition at the moving interface is continuity of the flux across the boundary, i.e., $J^{-}(h(t), t)=J^{+}(h(t), t)$. The quantity $J(x, t)$ is the flux of material at a point $x$ at time $t$ and the

$\$$ One might be tempted to assume that (3.2) should be of the form $C_{t}(x, t)=\frac{\partial}{\partial x}\left[D(x, t) C_{x}(x, t)\right]$, where $J(x, t) \equiv$ $-D(x, t) C_{x}(x, t)$ is the flux and, in the region $0<x<h(t), J(x, t)=-d_{1}(t-s(x))^{-1+\alpha} C_{x}(x, t)$. At the point $x=h(t), J$ is infinite by virtue of the identity $s(h(t)) \equiv t$ unless $C_{x}=0$, which is a severe restriction on the dynamics and would not guarantee conservation of flux across the interface. The flux condition corresponding to (3.2), to be derived below, is well-defined for all time. 
superscripts - and + denote the left and right sides of the interface, respectively. The flux relates to the diffusion equation in accordance with the equation (valid for 1D domains)

$$
C_{t}(x, t)=-\frac{\partial}{\partial x} J(x, t) ;
$$

this equation conserves mass. For SBM dynamics, the flux on the right is

$$
J^{+}(x, t)=-d_{2} t^{-1+\beta} C_{x}(x, t)
$$

so that, at the moving interface,

$$
J^{+}(h(t), t)=-d_{2} t^{-1+\beta} C_{x}\left(h^{+}(t), t\right) .
$$

Similarly, on the left, integration with respect to $x$ yields the flux

$$
J^{-}(x, t)=-\int_{-\infty}^{x} D\left(x^{\prime}, t\right) C_{x x}\left(x^{\prime}, t\right) d x^{\prime} .
$$

At $x=h(t)$, the condition reads

$$
J^{-}(h(t), t)=-\int_{-\infty}^{h(t)} D\left(x^{\prime}, t\right) C_{x x}\left(x^{\prime}, t\right) d x^{\prime} .
$$

Note that, although the flux condition contains an integral over the central domain, the past values of the concentration field do not appear in any of the equations. In other words, the SBM model lacks the history convolution associated with FAD dynamics. This distinction between SBM and FAD will become important in the numerical solutions to the problems. The flux condition, equation (3.12), does contain an integral over the central spatial domain, so numerical solutions are more computationally expensive to obtain than for the corresponding classical diffusion problem.

Additional boundary data are needed at the point $x=0$ due to the fact that he quantity $s(x)$ is piecewise-continuous there. At this point, there is an intersection of glassy material from the initial condition (where $s(x) \equiv 0$ ) and glassy material created by the propagation of the glass transition front (where $s(x)>0$ and $s(x) \rightarrow 0$ as $x \rightarrow 0^{+}$). At this stationary interface between original and newly-created material, continuity of the interface

$$
C\left(0^{-}, t\right)=C\left(0^{+}, t\right)
$$

and the flux

$$
C_{x}\left(0^{-}, t\right)=C_{x}\left(0^{+}, t\right)
$$

is imposed. Additionally, using the piecewise definition of $s(x)$, the flux at the left side of the moving interface (i.e., equation (3.12)) can be expressed as

$$
J^{-}(h(t), t)=-d_{1} t^{-1+\alpha} C_{x}\left(0^{-}, t\right)-d_{1} \int_{0}^{h^{-}(t)}\left(t-s\left(x^{\prime}\right)\right)^{-1+\alpha} C_{x x}\left(x^{\prime}, t\right) d x^{\prime} .
$$

Generalizations to more complex interface dynamics are straightforward; details will only be given if needed for clarification of the presentation. We now summarize the moving boundary problem on the infinite domain for SBM. The generalized diffusion dynamics is described by equations (3.2), (3.3) with initial and boundary data as in equations (3.4)-(3.7), (3.10), (3.13), (3.14), and (3.15). 


\section{A similarity solution: SBM with $\beta=\alpha$}

In the case that the two phases have the same order $(\beta=\alpha)$, a similarity solution of the moving boundary problem can be sought. In analogy to the classical similarity solution [26], suppose that the concentration field depends only on the similarity variable

$$
\eta=x / t^{\alpha / 2}
$$

and that the interface position depends on time as

$$
h(t)=\lambda t^{\alpha / 2} .
$$

Installing these assumptions into the PDE equations (3.2) and (3.3) and boundary data equations (3.4)-(3.7), (3.10), (3.13), (3.14), and (3.15) yields the ODE problem

$$
\left\{\begin{array}{lr}
C^{\prime \prime}(\eta)+\frac{\alpha}{2 d_{1}} \eta C^{\prime}(\eta)=0, & -\infty<\eta<0 \\
C^{\prime \prime}(\eta)+\frac{\alpha}{2 d_{1}} \eta\left(1-(\eta / \lambda)^{2 / \alpha}\right)^{1-\alpha} C^{\prime}(\eta)=0, & 0<\eta<\lambda \\
C^{\prime \prime}(\eta)+\frac{\alpha}{2 d_{2}} \eta C^{\prime}(\eta)=0, & \lambda<\eta<\infty \\
C(-\infty)=0, & \\
C(\infty)=c^{0}, & \\
C\left(\lambda^{ \pm}\right)=c^{*} & \\
C\left(0^{-}\right)=C\left(0^{+}\right), & \\
C^{\prime}\left(0^{-}\right)=C^{\prime}\left(0^{+}\right), & \\
d_{1} C^{\prime}\left(0^{-}\right)-d_{2} C^{\prime}\left(\lambda^{+}\right)+d_{1} \int_{0^{+}}^{\lambda^{-}}\left(1-\left(\eta^{\prime} / \lambda\right)^{2 / \alpha}\right)^{-1+\alpha} C^{\prime \prime}\left(\eta^{\prime}\right) d \eta^{\prime}=0 . &
\end{array}\right.
$$

Again, this formulation assumes that the interface is moving to the right (corresponding to $\lambda>0$ ). The differential equations are all first-order ODEs for the quantities $C^{\prime}(\eta)$ and can be solved exactly. The concentration field is given by

$$
C(\eta)= \begin{cases}b c^{*} \operatorname{erfc}\left(\frac{-\sqrt{\alpha}}{2 \sqrt{d_{1}}} \eta\right), & -\infty<\eta<0 \\ b c^{*}\left(1+\sqrt{\frac{\alpha}{\pi d_{1}}} \int_{0}^{\eta} e^{-G\left(\eta^{\prime}\right)} d \eta^{\prime}\right), & 0<\eta<\lambda \\ c^{0}+\left(c^{*}-c^{0}\right) \operatorname{erfc}\left(\frac{\sqrt{\alpha}}{2 \sqrt{d_{2}}} \eta\right) / \operatorname{erfc}\left(\frac{\sqrt{\alpha}}{\left.2 \sqrt{d_{2}} \lambda\right),}, \lambda<\eta<\infty\right.\end{cases}
$$

where

$$
b=\left(1+\sqrt{\frac{\alpha}{\pi d_{1}}} \int_{0}^{\lambda} e^{-G\left(\eta^{\prime}\right)} d \eta^{\prime}\right)^{-1}
$$

and

$$
\begin{aligned}
G(\eta) & =\frac{\alpha}{2 d_{1}} \int_{0}^{\eta} \eta^{\prime}\left(1-\left(\eta^{\prime} / \lambda\right)^{2 / \alpha}\right)^{1-\alpha} d \eta^{\prime} \\
& =\frac{\alpha^{2} \lambda^{2}}{4 d_{1}}\left(B_{(\eta / \lambda)^{2 / \alpha}}(\alpha, 1-\alpha)-B_{(\eta / \lambda)^{2 / \alpha}}(1+\alpha, 1-\alpha)\right) .
\end{aligned}
$$

The expression $B_{z}(a, b)$ is the incomplete Beta function. The front position $\lambda$ is determined from the flux condition, which yields the transcendental equation

$$
\left(1-\frac{\alpha}{2 d_{1}} \int_{0}^{\lambda} \eta^{\prime} e^{-G\left(\eta^{\prime}\right)} d \eta^{\prime}\right) e^{\frac{\alpha}{d_{2}} \lambda^{2}} \operatorname{erfc}\left(\frac{\sqrt{\alpha}}{2 \sqrt{d_{2}}} \lambda\right)-\frac{1}{b} \sqrt{\frac{d_{2}}{d_{1}}} \frac{c^{0}-c^{*}}{c^{*}}=0 .
$$


In the case that the interface is moving to the left, the concentration field is

$$
C(\eta)= \begin{cases}c^{*} \operatorname{erfc}\left(\frac{-\sqrt{\alpha}}{2 \sqrt{d_{1}}} \eta\right) / \operatorname{erfc}\left(\frac{-\sqrt{\alpha}}{2 \sqrt{d_{1}}} \lambda\right), & -\infty<\eta<\lambda, \\ c^{0}+\tilde{b}\left(c^{*}-c^{0}\right)\left(1+\sqrt{\frac{\alpha}{\pi d_{2}}} \int_{\eta}^{0} e^{G\left(\eta^{\prime}\right)} d \eta^{\prime}\right), & \lambda<\eta<0, \\ c^{0}+\tilde{b}\left(c^{*}-c^{0}\right) \operatorname{erfc}\left(\frac{\sqrt{\alpha}}{2 \sqrt{d_{2}}} \eta\right), & 0<\eta<\infty,\end{cases}
$$

where

$$
\tilde{b}=\left(1+\sqrt{\frac{\alpha}{\pi d_{2}}} \int_{\lambda}^{0} e^{G\left(\eta^{\prime}\right)} d \eta^{\prime}\right)^{-1}
$$

and $G(\eta)$ is defined as above with $d_{1} \rightarrow d_{2}$. In this case, the transcendental equation for $\lambda$ is

$$
\left(1+\frac{\alpha}{2 d_{2}} \int_{\lambda}^{0} \eta^{\prime} e^{G\left(\eta^{\prime}\right)} d \eta^{\prime}\right) e^{\frac{\alpha}{4 d_{1}} \lambda^{2}} \operatorname{erfc}\left(\frac{-\sqrt{\alpha}}{2 \sqrt{d_{1}}} \lambda\right)-\frac{1}{\tilde{b}} \sqrt{\frac{d_{1}}{d_{2}}} \frac{c^{*}}{c^{0}-c^{*}}=0 .
$$

For the case of a stationary front (i.e., $\lambda=0$ ), equations (4.8) and (4.11) reduce to the algebraic equation

$$
\sqrt{\frac{d_{2}}{d_{1}}}=\frac{c^{*}}{c^{0}-c^{*}} .
$$

This parameter-space curve separates the regions in which the leftward-moving and rightwardmoving formulations are valid. Interestingly, the dependence upon the anomalous diffusion parameter $\alpha$ vanishes in this limit.

Figure 2 shows the similarity solution in comparison to the FAD and classical models for $\alpha=$ $0.8, c^{*}=0.6, c^{0}=1$, and $d_{1}=d_{2}=1$ at various times. The diffusivities for the SBM solutions are adjusted by a factor of $1 / \Gamma(\alpha)$ (see equation (2.13)). The FAD result is a numerical solution of the corresponding problem, as solved in [24], and the classical result is obtained by taking the limit $\alpha \rightarrow 1$ in the similarity solution. The left panel shows the concentration fields for these three models for times $t=0.01, t=0.1, t=1.0, t=10.0$, and $t=100.0$. There is good agreement between the SBM and FAD results for all five time values. The classical result differs significantly for small time due to the fact that the classical problem lacks the time-dependent coefficient in the SBM model, which is singular at $t=0$. As time approaches 1, the classical and SBM models are governed by the same equation in the left and right (but not central) regions of the domain, so the solutions are close at this time. For larger time, the classical solution overtakes the two anomalous concentration fields. This phenomenon can be understood by considering the two interface time scalings $t^{1 / 2}$ and $t^{\alpha / 2}$; when $t<1, t^{1 / 2}<t^{\alpha / 2}$ and vice versa for $t>1$. The right panel of this figure is a plot of the interface position $h(t)$ for these three models; the SBM and FAD models have the same time dependence with a different coefficient, $h(t)=\lambda t^{\alpha / 2}$. The interface position in the classical result scales as $h(t)=\lambda \sqrt{t}$, indicating that the classical interface lags behind the SBM and FAD results for small time but advances faster for large time. These observations agree with the assumption of similarity in the three models.

A natural question that arises about this similarity result concerns its stability with respect to arbitrary but small spatial perturbations to the initial condition. An analytical linear stability analysis of the system is difficult due to the integrable singularity in the flux condition (the last equation in (4.3)). This integrable singularity yields singular integrals in higher-order problems when the 

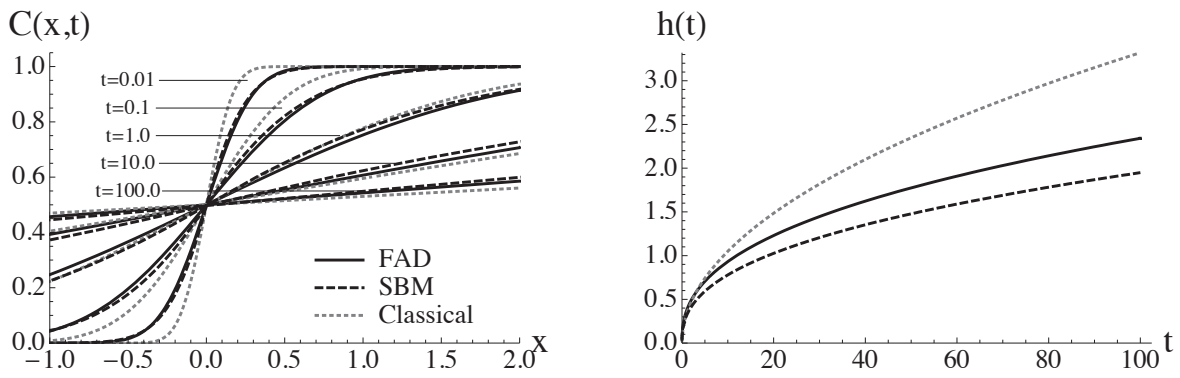

FIG. 2. Left: the concentration field for FAD (solid), SBM (dashed), and classical (dotted) models at times $t=0.01$, $t=0.1, t=1.0, t=10.0$, and $t=100.0$. Right: plot of the interface position $h(t)$ for these three models; the SBM and FAD models have the same time dependence with a different coefficient. In the figure, $\alpha=0.8, c^{*}=0.6, c^{0}=1$, and $d_{1}=d_{2}=1$. The diffusivities for the SBM solutions are adjusted by a factor of $1 / \Gamma(\alpha)$.

quantity $s(x)$ is perturbed. Due to this complication, a numerical stability analysis is conducted instead. The Heaviside initial condition is disturbed by the addition of an $\mathrm{O}(\delta)$ (where $0<\delta \ll 1$ ) function. The location and functional form of this disturbance are varied, as are the parameters $\alpha$, $d_{1}, d_{2}$, and $c^{*}$. Irrespective of the parameter values, the perturbation to the initial condition decays as the computation progresses, and the solution converges to the similarity solution reported above. Consequently, there is evidence that the similarity solution is stable to disturbances, and this stability is unaffected by the choice of the parameter values in the computation. Although these results imply stability, there is no conclusive evidence that all such disturbances decay due to the fact that the investigation is computational rather than analytical. It should be noted that, in the corresponding classical diffusion $(\alpha=1)$ problem, an analytical linear stability analysis reveals that the similarity solution is stable for all values of the parameters [14].

A similarity solution can also be found in the semiinfinite problem in which the concentration is held at $c^{0}>c^{*}$ at $x=0$, the far-field concentration is 0 at $x \rightarrow \infty$, and the initial concentration is zero for $x>0$. The boundary conditions at the interface are the same. In this case, the anomalous region is on the right and the lower limit of integration is $s(x)=0$. Also, note that the similarity solutions in equations (4.4) and (4.9) recover their classical counterparts in the limit that $\alpha \rightarrow 1$, as expected.

\section{Vitrification models}

\section{$5.1 \quad$ Numerical solution for one SBM and one classical diffusion domain}

Another interesting class of problems involving scaled Brownian motion is the situation in which only one domain exhibits subdiffusion dynamics, the other region being classical in nature. Due to the different time scalings between the two regions, there is no similarity solution. The model obeys the problem given by the PDE equations (3.2) and (3.3) and boundary data equations (3.4)-(3.7), (3.10), (3.13), (3.14), and (3.15) with $\beta=1$ and $\alpha<1$. Due to the lack of similarity, the interface does not scale as $h(t)=\lambda t^{\alpha / 2}$ nor as $h(t)=\lambda \sqrt{t}$. The problem is solved using finite difference methods; the algorithm is described briefly in the following paragraph and is examined in much more detail in [14].

This problem is solved numerically using an implicit finite difference scheme with first-order backward differencing in time and second-order central differencing in space. Far from the interface, 
the grid points are updated using a typical (1-2) implicit scheme. Within two grid points of the interface, however, the update scheme requires special treatment since the moving interface propagates amid a fixed spatial discretization. The grid points just to the left and right of the interface are temporarily ignored while the interface position is updated by means of an iterative technique. Since the interface typically lies between two grid points, the grid points to its left and right (after ignoring the points in the immediate vicinity of the interface) are discretized using non-centred differencing. The interface location is then updated according to the following iterative approach: using the previous interface position as an ansatz, the concentration field is computed and a new interface position is calculated using this concentration solution. The new interface position is then compared to the ansatz and, based on a tolerance parameter, is either accepted as the new position or used as the ansatz in the next iteration. Once the interface position converges sufficiently, the points just to the left and right of the interface are computed using non-centred differencing.

Plots of the concentration field are not provided due to their close resemblance to the solutions illustrated in Figure 2 for the similarity solution. The position of the interface $h(t)$ is shown for various parameter values in Figures 3 and 4. These data reveal an interesting phenomenon: the interface actually turns around in the FAD and SBM models after it advances for a time. This behavior was first noticed in numerical solutions of the corresponding FAD system in [24]; in the case of SBM, we find that the same feature emerges. The turnaround phenomenon in the SBM system can be understood by appealing to the effective diffusivity in the anomalous region, equation (3.3). For small time, $d_{1} t^{-1+\alpha}$ is large; as a result, material which crosses over the interface into the anomalous region quickly diffuses farther into the domain. This behavior impedes the accumulation of material in the vicinity of the interface, facilitating the propagation of the interface in the rightward direction. We note that the interface always moves to the right initially, irrespective of parameter values, due to this singularity. Conversely, for large time, this effective diffusivity is small, which causes an accumulation of material on the left side of the interface. Eventually, this effect is significant enough that there is no longer a negative slope just to the left of the interface and the moving boundary reverses direction.

Figure 3 exhibits this turnaround behavior and its dependence on the critical concentration $c^{*}$ for both SBM and FAD with parameters $\alpha=0.8, \beta=1, d_{1}=d_{2}=1$, and $c^{0}=1$. As the value of $c^{*}$ is lowered, the turnaround time approaches 0 due to the fact that there is an increasingly large propensity for the classical diffusion region to "win out" in this limit. The interface eventually turns to the left due to the diminishing effective diffusivity in the anomalous region for large time (i.e., since the effective diffusivity scales as $D(x, t) \sim t^{-1+\alpha}$ in the anomalous regime). Note that the figure shows $c^{*}$ values in the range $0.62 \leqslant c^{*} \leqslant 0.7$. This choice is purely aesthetic; this range of values demonstrates the existence of short and long turnaround times. The numerical algorithm is capable of producing results for the full range $0<c^{*}<1$. In the corresponding classical diffusion problem, when the diffusivities are equal and $c^{0}=1$, the interface moves right when $c^{*}>0.5$, is stationary when $c^{*}=0.5$, and moves to the left when $c^{*}<0.5$.

Figure 4 exhibits the dependence of the turnaround time on the anomalous diffusion order $-1+\alpha$ with parameters $\beta=1, d_{1}=d_{2}=1, c^{*}=0.7$, and $c^{0}=1$. As the value of $\alpha$ is lowered (i.e., as the dynamics of the subdiffusive region deviates more from classical diffusion behavior), the turnaround time approaches 0 . This observation can also be explained in accordance with the effective diffusivity: although $d_{1} t^{-1+\alpha}$ has a stronger singularity for small time as the value of $\alpha$ is reduced (causing the anomalous region to grow), its value also approaches 0 more quickly as time is increased (causing the interface to reverse direction). Consequently, the propensity for the 


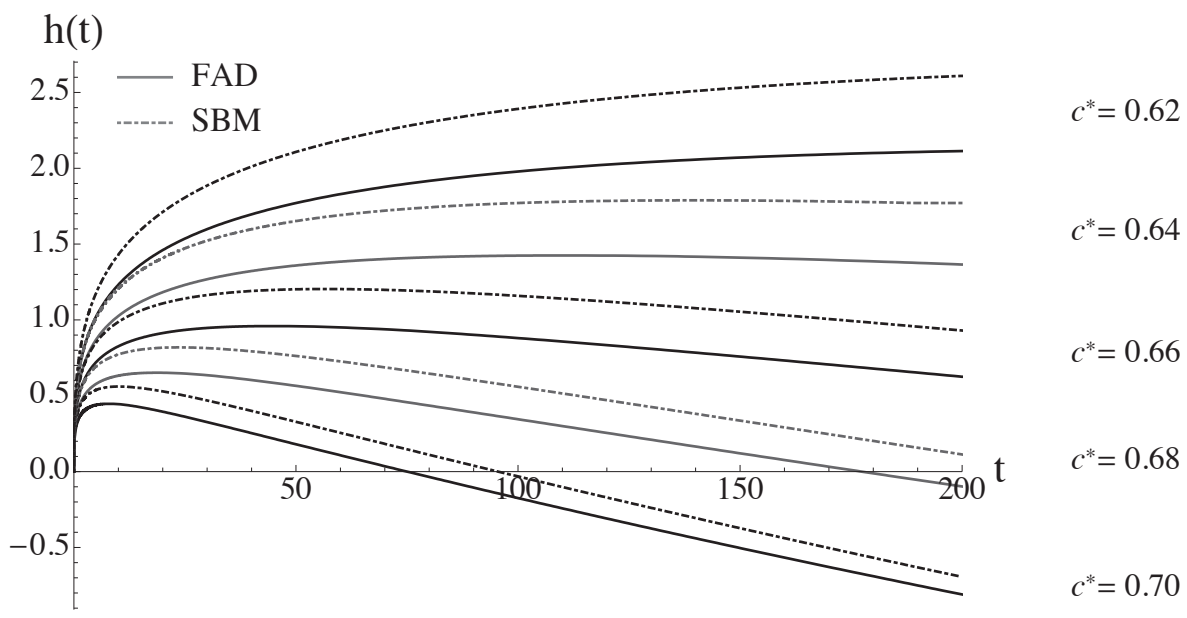

FIG. 3. Investigation of the turnaround phenomenon as the critical concentration $c^{*}$ is varied. The SBM and FAD results match well, particularly when the turnaround time is small. In the figure, $\alpha=0.8, \beta=1, d_{1}=d_{2}=1$, and $c^{0}=1$.

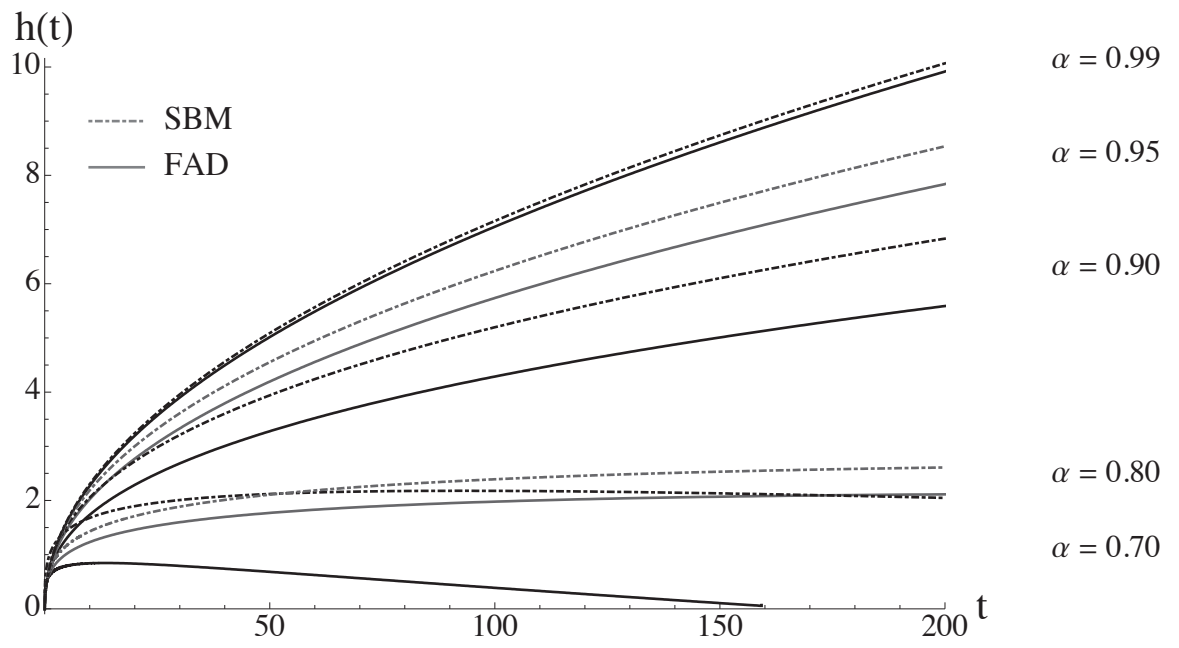

FIG. 4. Investigation of the turnaround phenomenon as the anomalous parameter $\alpha$ is varied. The SBM and FAD results match well in general, though the agreement is not as good when the turnaround time is large. Nevertheless, the two models converge in the limit as $\alpha \rightarrow 1$. In the figure, $\beta=1, d_{1}=d_{2}=1, c^{*}=0.7$, and $c^{0}=1$

SBM region to overtake the classical region diminishes more quickly for smaller $\alpha$. As expected, the solutions converge in the limit that $\alpha \rightarrow 1$. Note that the range of the anomalous parameter in the plot is $0.7 \leqslant \alpha \leqslant 1$. The restrictive lower bound on this range is a result of the fact that the turnaround time quickly approaches zero as $\alpha$ is taken lower. It becomes difficult to resolve the dynamics numerically for the case of small $\alpha$.

The turnaround time, $t^{*}$, is investigated by computing the solution over a range of parameter values for $c^{*}$ and $\alpha$ and fitting the resulting data to an ansatz matching function. Based on the 


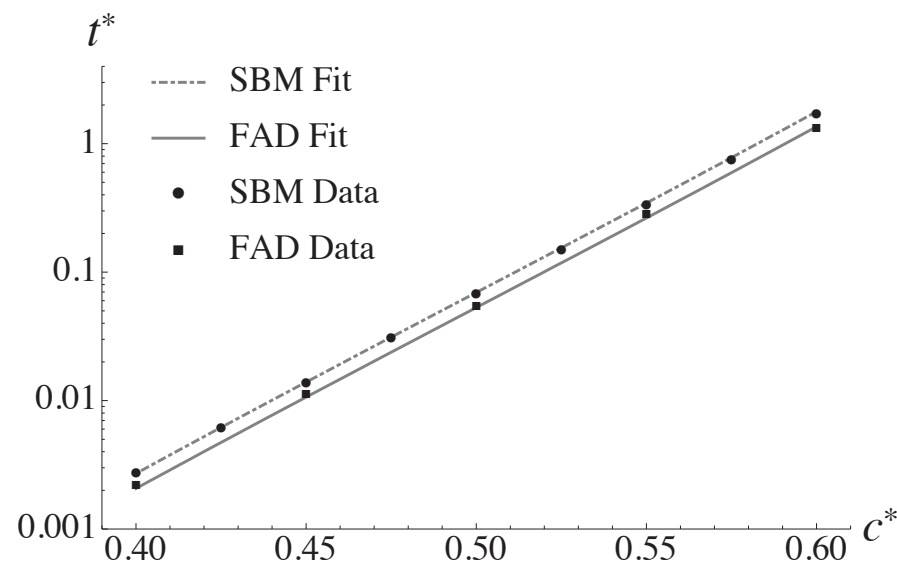

FIG. 5. Comparison between the ansatz functions 5.3 and 5.4 and the simulation data on which the fitting parameters were determined. There is good agreement between simulation data and the fitting functions.

corresponding parameter dependence for the FAD results [24], suppose that the turnaround time has the form

$$
t^{*}=f(\alpha)\left(\frac{c^{*}}{1-c^{*}}\right)^{\frac{2}{1-\alpha}} .
$$

The turnaround time is determined for the parameter ranges $c^{*} \in[0.4,0.6]$ and $\alpha \in[0.7,0.9]$ and numerically fitted to the ansatz above. For each alpha, the quantity

$$
f(\alpha)=t^{*}\left(\frac{c^{*}}{1-c^{*}}\right)^{-\frac{2}{1-\alpha}}
$$

is averaged over all the $c^{*}$ values. The data for the coefficient $f(\alpha)$ exhibited a nonlinear dependence on $\alpha$. Fitting the data for this coefficient, the expected behavior of the turnaround time is

$$
t^{*}=0.11 \alpha^{1.6}\left(\frac{c^{*}}{1-c^{*}}\right)^{\frac{2}{1-\alpha}} .
$$

For comparison, the fit for FAD is [24]

$$
t^{*}=0.078 \alpha^{1.3}\left(\frac{c^{*}}{1-c^{*}}\right)^{\frac{2}{1-\alpha}} .
$$

Figure 5 is an overlay of these fitting functions and the data on which they are based. There is good agreement between the simulation data and the fitting ansatz functions.

Similarly, the turnaround location, $h^{*}$, is explored using the same data and fitting to an ansatz. Suppose that the turnaround location has the form

$$
h^{*}=\theta(\alpha)\left(\frac{c^{*}}{1-c^{*}}\right)^{\kappa(\alpha)},
$$

where

$$
\theta(\alpha)=a \frac{1-\alpha}{\alpha}+b
$$


and

$$
\kappa(\alpha)=k \frac{1}{1-\alpha}+l
$$

Using the same parameter ranges as for $t^{*}$, the parameters of the ansatz are determined. Fitting the data, the expected behavior of the turnaround location is

$$
h^{*}=\left(0.24 \frac{1-\alpha}{\alpha}+0.0048\right)\left(\frac{c^{*}}{1-c^{*}}\right)^{0.951 \frac{1}{1-\alpha}-0.670} .
$$

For comparison, the fit for FAD is [24]

$$
h^{*}=\left(0.20 \frac{1-\alpha}{\alpha}+0.0020\right)\left(\frac{c^{*}}{1-c^{*}}\right)^{1.01 \frac{1}{1-\alpha}-0.732}
$$

It is important to remark again that this turnaround phenomenon is mirrored in numerical solutions of the problem where FAD replaces SBM. In other words, even though there are quantitative differences in the interface position between the two models, the qualitative behavior is the same (this observation can be seen in Figures 3 and 4). This observation motivates the next section, which focuses on the use of the SBM model as a replacement for FAD. The SBM model has clear computational advantages over FAD due to its lack of a convolution integral in the governing equation. Consequently, its use as a model for subdiffusion processes is desirable.

Table 1 compares the run times of the SBM and FAD numerical solution code for $10^{2}, 10^{3}$, and $10^{4}$ timesteps. The two solution codes use an identical algorithm; they differ only in the governing PDEs and flux condition. In all cases, both the FAD and SBM runs used a spatial step size of $\Delta x=7.5 \cdot 10^{-3}$ and a temporal step size of $\Delta t=10^{-5}$. As the run times demonstrate, each tenfold increase in the number of timesteps causes the run time of the FAD code to increase by approximately one hundred. In other words, the FAD code entails an $\mathrm{O}\left(N^{2}\right)$ computation; this scaling is to be expected due to the fact that the fractional PDE contains a convolution integral in time. On the other hand, the run time for the SBM code increases by very roughly a factor of ten with a tenfold increase in the number of timesteps, indicating that the SBM code is $\mathrm{O}(N)$. The increase in run time is somewhat higher than a factor of ten due to the form of the flux condition. In the implementation of the numerical solution, continuity of flux is imposed through equation (3.15). This formulation contains an integral over the spatial domain $x \in[0, h(t)]$; the size of this domain is 0 initially and grows until the point at which the interface reverses direction. As a result, the scaling in run time for the SBM numerical solution code is somewhat larger than $\mathrm{O}(N)$. The discrepancy between the scaling of computational times between the two codes highlights the primary advantage of using SBM as a model for subdiffusive phenomena. This difference in run time becomes particularly important in higher-dimensional problems.

\subsection{Numerical solution for two SBM regions}

A generalization of the vitrification model from above is the situation in which a moving interface separates two anomalous regions with differing orders; this situation is illustrated in Figure 1. This more general problem corresponds to the original problem statement. Without loss of generality, take $\alpha<\beta<1$. This problem is solved numerically using the same algorithm as in the case of $\alpha<1, \beta=1$. 
TABLE 1. Comparison of Run Times for SBM and FAD Models

\begin{tabular}{ccc}
\hline Timesteps & SBM Run Time (s) & FAD Run Time (s) \\
\hline $10^{2}$ & 0.24 & 11.79 \\
$10^{3}$ & 3.05 & $1,200.98$ \\
$10^{4}$ & 116.07 & $118,170.69$ \\
\hline
\end{tabular}

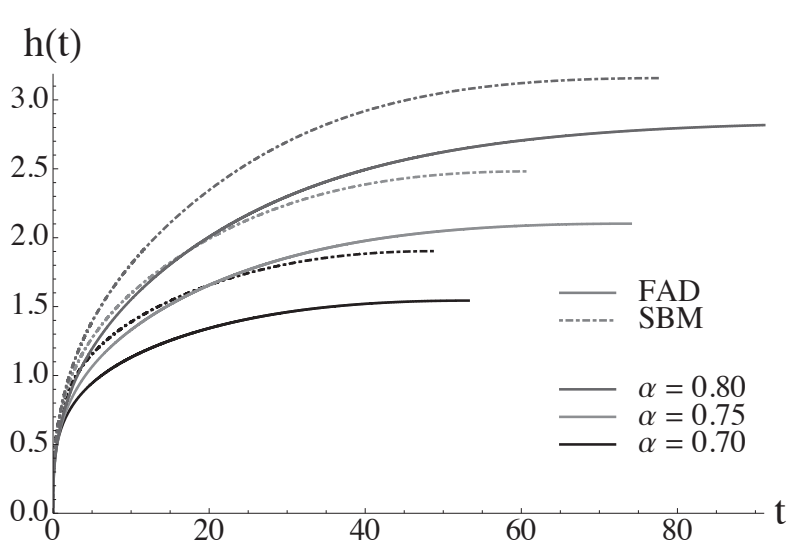

FIG. 6. Investigation of the turnaround phenomenon as the anomalous parameter $\alpha$ is varied for $\beta=0.85$ fixed. In the figure, $d_{1}=d_{2}=1, c^{*}=0.7$, and $c^{0}=1$.

Figure 6 is analogous to Figure 4 for the case of $\beta=0.85$ (keeping the other parameter values the same). The parameter $\alpha$ is varied, and the resulting dynamics spans the range from short turnaround to large turnaround. The solutions to this problem are phenomenologically the same as those for the case of $\beta=1, \alpha<1$. This resemblance can be understood once again by appealing to the effective diffusivity, which is "more singular" on the left than on the right, causing the same qualitative behavior to emerge.

\section{Asymptotic solution for nearly-classical problem}

In a prior section, an asymptotic expansion of the Riemann-Liouville operator was developed (equation (2.20)). The leading-order behavior of the operator was shown to have the form of an SBM process. In this section, we consider a problem on the positive semiaxis (discussed briefly above) with classical diffusion on the left and SBM dynamics with order $-1+\alpha$ on the right. Since there is a domain boundary at $x=0$, the interface can only move to the right, so that the classical region overtakes the anomalous region (in the context of the vitrification problems introduced at the beginning of the paper, this situation corresponds to the reconstitution of a vitrified material). In this case, we take $\alpha=1-\epsilon$, where $0<\epsilon \ll 1$, so that the anomalous behavior is nearly-classical. The solution of this problem gives insight into the usefulness of using SBM in place of FAD in the case of nearly-classical behavior in the anomalous region (i.e., when the order $-1+\alpha$ is near 0 ). 
In [14], it is shown that the flux condition for this nearly-classical problem has the form

$$
d_{1} C_{x}\left(h^{-}(t), t\right)=d_{2} t^{-\epsilon} C_{x}\left(h^{+}(t), t\right) .
$$

To solve the initial-boundary-value problem, change variables to the similarity variable from the classical problem, $\eta=x / \sqrt{t}$, and the timescale $\tau=t^{\epsilon}$ and suppose that the interface position has the form $h(t)=\sqrt{t} \lambda(\tau)=\tau^{\frac{1}{2 \epsilon}}\left(\lambda_{0}(\tau)+\mathrm{O}(\epsilon)\right) \sim \tau^{\frac{1}{2 \epsilon}} \lambda_{0}(\tau)$. Let $C(x, t)=C(\eta, \tau)$ and expand the concentration field as $C(\eta, \tau)=C_{0}(\eta, \tau)+\epsilon C_{1}(\eta, \tau)+\mathrm{O}\left(\epsilon^{2}\right)$ so that the leading-order problem can be formulated as

$$
\left\{\begin{array}{lll}
C_{0, \eta \eta}(\eta, \tau)+\frac{1}{2 d_{1}} \eta C_{0, \eta}(\eta, \tau)=0, & \tau>0, & 0<\eta<\lambda_{0}(\tau), \\
C_{0, \eta \eta}(\eta, \tau)+\frac{1}{2 d_{2}} \tau \eta C_{0, \eta}(\eta, \tau)=0, & \tau>0, & \lambda_{0}(\tau)<\eta<\infty, \\
C_{0}(0, \tau)=c^{0}, & \tau>0, & \\
C_{0}(\infty, \tau)=0, & \tau>0, \\
C_{0}(\eta, 0)=0, & \eta>0, \\
C_{0}\left(\lambda_{0}(\tau), t\right)=c^{*}, & \tau>0, \\
d_{1} C_{0, \eta}\left(\lambda_{0}^{-}(\tau), \tau\right)=d_{2} \frac{1}{\tau} C_{0, \eta}\left(\lambda_{0}^{+}(\tau), \tau\right), & \tau>0 .
\end{array}\right.
$$

Note that a term proportional to $\epsilon \tau C_{\tau}(\eta, \tau)$ is discarded, which converts the leading-order problem into an ODE in the variable $\eta$. The elimination of this term is justified on the grounds that the product $\tau C_{\tau}(\eta, \tau)=\mathrm{O}(1)$, indicating that the term is $\mathrm{O}(\epsilon)$. Solving the ODE using elementary techniques and imposing the boundary conditions, the leading-order solution for the concentration field is

$$
\begin{cases}C_{0}(\eta, \tau)=c^{0}+\left(c^{*}-c^{0}\right) \operatorname{erf}\left(\frac{1}{2 \sqrt{d_{1}}} \eta\right) / \operatorname{erf}\left(\frac{1}{2 \sqrt{d_{1}}} \lambda_{0}(\tau)\right), & 0<\eta<\lambda_{0}(\tau), \\ C_{0}(\eta, \tau)=c^{*} \operatorname{erfc}\left(\frac{\sqrt{\tau}}{2 \sqrt{d_{2}}} \eta\right) / \operatorname{erfc}\left(\frac{\sqrt{\tau}}{2 \sqrt{d_{2}}} \lambda_{0}(\tau)\right), & \lambda_{0}(\tau)<\eta<\infty .\end{cases}
$$

The final piece of boundary data is the flux condition, which yields an equation for $\lambda_{0}(\tau)$. Imposing this condition and rearranging,

$$
\frac{1}{\sqrt{\tau}} e^{-\frac{1}{4}\left(\lambda_{0}(\tau)\right)^{2}\left(\frac{\tau}{d_{2}}-\frac{1}{d_{1}}\right)} \operatorname{erf}\left(\frac{1}{2 \sqrt{d_{1}}} \lambda_{0}(\tau)\right) / \operatorname{erfc}\left(\frac{\sqrt{\tau}}{2 \sqrt{d_{2}}} \lambda_{0}(\tau)\right)-\sqrt{\frac{d_{1}}{d_{2}}} \frac{c^{0}-c^{*}}{c^{*}}=0 .
$$

The concentration field solution (equation (6.3)) and the transcendental equation for $\lambda_{0}(\tau)$ (equation (6.4)) reduce to their classical counterparts in the limit that $\epsilon \rightarrow 0$ (which corresponds to $\tau \rightarrow 1$ ). The concentration field is plotted in comparison to the other models for three different times in the left panel of Figure 7. The results for the leading-order solution, the numerical solution of the SBM model, and the corresponding FAD problem agree very well and all three differ from the classical concentration field, particularly for small time.

In previous problems, the flux condition yielded a transcendental equation for the constant $\lambda$. In the present case, FAD with derivative order $\epsilon$, equation (6.4), can be considered a continuum of transcendental equations for $\lambda_{0}(\tau)$, one for each value of $\tau$. Equation (6.4) is solved numerically and plotted in the right panel of Figure 7. There are two regions of interest: for small $\tau, \lambda_{0}(\tau)$ scales as $\sqrt{\tau}$, while, for large $\tau, \lambda_{0}(\tau)$ levels off to a constant value. These observations can be verified using the known asymptotic expansions of the special functions in (6.4). For large $\tau$, employ the large-argument asymptotics of $\operatorname{erfc}(x)$,

$$
\operatorname{erfc}(x) \sim \frac{1}{\sqrt{\pi}} \frac{1}{x} e^{-x^{2}}
$$


the $\tau$-dependence drops out, yielding a transcendental equation for constant $\lambda_{0}$ :

$$
\lambda_{0} \operatorname{erf}\left(\frac{1}{2 \sqrt{d_{1}}} \lambda_{0}\right) e^{\frac{\lambda_{0}^{2}}{4 d_{1}}}-2 \sqrt{\frac{d_{1}}{\pi}} \frac{c^{0}-c^{*}}{c^{*}}=0 .
$$

For small $\tau$, the only matching consistent with the equation is $\lambda_{0}(\tau) \sim a \sqrt{\tau}$. Using this expression as well as the small-argument expansions

$$
\begin{gathered}
\operatorname{erf}(x) \sim \frac{2}{\sqrt{\pi}} x, \\
\operatorname{erfc}(x) \sim 1,
\end{gathered}
$$

and

$$
e^{x} \sim 1
$$

equation (6.4) reduces to an algebraic equation for the coefficient $a$. The small- $\tau$ behavior of $\lambda_{0}(\tau)$ is

$$
\lambda_{0}(\tau) \sim a \sqrt{\tau}=d_{1} \sqrt{\frac{\pi}{d_{2}}} \frac{c^{0}-c^{*}}{c^{*}} \sqrt{\tau} .
$$

The concentration-field solution (equation (6.3)) is plotted in the left panel of Figure 7 at times $t=10^{-4}, t=10^{-2}, t=10^{0}$, and $t=10^{2}$ on semilog axes and with parameter values $\alpha=0.9$, $d_{1}=d_{2}=3, c^{*}=0.25$, and $c^{0}=1$. Note that the concentration is continuous at the point of the phase transition front, but the slope is discontinuous. This observation is rooted in the fact that the diffusivity is effectively a function of time in the SBM region. In classical problems, there is a discontinuous slope at the interface due to continuity of the flux across the interface (this feature is absent here due to the fact that $d_{1}=d_{2}$ in this case). It is important to note that the leadingorder solution developed in this section matches well with the other two (numerical) anomalous diffusion solutions. The numerical solution of the FAD model is not reported for $t=10^{2}$ due to computational expense. The numerical solution of the "transcendental equation" (6.4) is plotted in the right panel of Figure 7 on logarithmic axes. For comparison, the small- and large- $\tau$ asymptotics are included. The very close agreement between the numerical solution (discrete points) and the asymptotic scalings verifies that the moving front has two distinct behavioral regimes in the smalland large- $\tau$ limits. The numerical interface position for the SBM model is also plotted. The solutions agree well up to time $10^{2}$, as demonstrated in the inset plot.

For small $\tau$, the interface scales as

$$
h(t) \sim \sqrt{t} \lambda_{0}(\tau) \sim d_{1} \sqrt{\frac{\pi}{d_{2}}} \frac{c^{0}-c^{*}}{c^{*}} t^{(1+\epsilon) / 2} .
$$

This behavior indicates that the propagation of the interface is slower than for classical diffusion since $t$ is small in this limit. The cause of this behavior lies in the effective diffusivity. In the previous models, the singularity in the effective diffusivity in the SBM region at small time forced the interface to propagate into the classical region (or, in the case of two SBM regions, in the direction of the less anomalous regime). In this geometry, the interface can only propagate into the anomalous region due to the domain boundary at $x=0$. As a result, the singularity in the effective diffusivity, which quickly carries material away from the interface and into the anomalous 

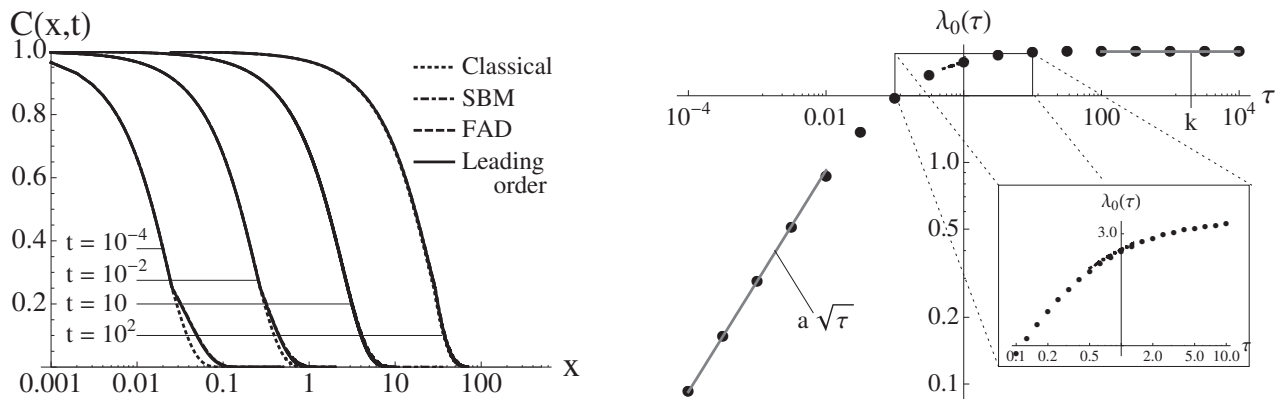

FIG. 7. Left: comparison of the concentration fields of the leading-order (analytical), SBM (numerical), FAD (numerical), and classical (analytical) solutions at four times. The FAD model is not reported for $t=10^{2}$ due to computational expense. Right: plot of the computed solution of $\lambda_{0}(\tau)$. This numerical solution and the asymptotic results agree well. Also shown is the interface location for the SBM problem $\left(\lambda_{0}(\tau)=h(t) / \sqrt{t}\right)$; the inset shows the SBM solution in more detail. In the figure, $\alpha=0.9, d_{1}=d_{2}=3, c^{*}=0.25$, and $c^{0}=1$.

domain, restricts the propagation of the interface rightward. In other words, in the small-time limit, the dynamics of the interface propagation is subdiffusion-dominated.

For large $\tau$, the interface scales as $h(t) \sim \lambda_{0} \sqrt{t}$. It is important to note that the transcendental equation for $\lambda_{0}$ (equation (6.6)) is not the transcendental equation for $\lambda$ in the classical case (equation (4.8) with $\alpha=1$ ). Consequently, this coefficient, and hence the position of the interface, can either lag or lead the analogous classical interface position, depending on parameter values.

\section{Conclusions}

Our objective in this paper is to investigate the scaled Brownian motion (SBM) model of subdiffusion in application problems. Particular attention is paid to the relationship of this model to a more established model of subdiffusion, fractional anomalous diffusion (FAD). We find that, in general, the solutions for the two models are qualitatively identical, though there can be large quantitative differences in some parameter regimes. The agreement between the SBM and FAD solutions raises an important question about the modeling of subdiffusive phenomena. In practice, experimental setups are designed to measure the mean-squared displacement of particles in subdiffusive media; since both models exhibit the same time dependence in their mean-squared displacements, many materials cannot be unambiguously characterized as SBM or FAD systems. As a result, in the absence of further experimental data (e.g., interface position as a function of time), either model can be employed to predict the behavior of a system. As a result of the investigations reported in this paper, we contend that, for subdiffusive systems uncommitted to a particular model, SBM is a superior to FAD due to the relative ease with which SBM solutions can be computed.

Section 4 investigates a similarity problem with two anomalous diffusion regions. The timescale $t^{\alpha / 2}$ emerges, in analogy to the classical $t^{1 / 2}$ timescale. As in the classical case, an exact analytical solution to the problem can be obtained using elementary techniques.

Section 5 examines a problem in which the two regions have differing anomalous diffusion orders $(\alpha \neq \beta)$. In this situation, the interface always propagates in such a way that the region with the smaller order grows initially and, after a time, the interface reverses direction. This turnaround phenomenon is explained by the time dependence of the effective diffusivity in the SBM model. 
In the SBM regions, $D(x, t)=d_{1}(t-s(x))^{-1+\alpha}$; this effective diffusivity has a singularity at $t=0$ since $\alpha$ obeys $0<\alpha<1$. As a result, the diffusivity is effectively very large at small time and small at large time. Consequently, for small time, material entering the SBM region is quickly carried further into the domain and away from the interface, which forces propagation of the interface rightward. For large time, water accumulates near the interface and the moving boundary then reverses direction.

Finally, Section 6 investigate a problem with an FAD region of order $1-\epsilon$, where $0<\epsilon \ll 1$. This problem yields insight into the use of SBM as an approximation to FAD. The asymptotic expansion (2.20) is applied to the FAD operator and the resulting leading-order (SBM) problem is solved using the techniques of the similarity problem. SBM and FAD are qualitatively the same, as expected, and their quantitative agreement is good in the limit of small $\epsilon$.

The replacement of FAD problems with SBM is desirable due to the difficulty in solving problems that include fractional derivative operators. FAD problems are tractable (from the standpoint of analytical methods) only in certain simple geometries due to the memory property of the fractional operator. Computationally, these problems are also intensive to solve due to the convolution integral in the governing equation. The storage requirements and computational expense of computing a history at every timestep (an $\mathrm{O}\left(N^{2}\right)$ computation) for each spatial point can quickly make a 1D problem prohibitive. Consequently, the use of SBM is beneficial since the numerical solution of SBM problems is $\mathrm{O}(N)$ at each spatial grid point. These differences become especially relevant in multiple spatial dimensions.

\section{Acknowledgments}

This work was supported in part by NIH Grant 1R01GM086886 and NSF RTG Grant DMS0636574 .

\section{REFERENCES}

1. AKsan, A. \& TONER, M., Isothermal desiccation and vitrification kinetics of trehalose-dextran solutions. Langmuir 20 (2004), 5521-5529.

2. Aksan, A., Irimia, D., He, X. \& Toner, M., Desiccation kinetics of biopreservation solutions in microchannels. Journal of Applied Physics 99 (2006), 064703.

3. Berthier, L., Biroli, G., Bouchaud, J. P., Cipelletti, L., El Masri, D., L'Hôte, D., Ladieu, F. \& PERINO, M., Spontaneous and induced dynamic fluctuations in glass-formers I: General results and dependence on ensemble and dynamics. Science 310 (2005), 1997.

4. Chen, T., Acker, J. P., Eroglu, A., Cheley, S., Bayley, H., Fowler, A. \& Toner, M., Beneficial effect of intracellular trehalose on the membrane integrity of dried mammalian cells. Cryobiology 43 (2001), 168-181.

5. Chen, T., Bhowmick, S., Sputtek, A., Fowler, A. \& Toner, M., The glass transition temperature of mixtures of trehalose and hydroxyethyl starch. Cryobiology 44 (2002), 301-306.

6. Conrad, P. \& De Pablo, J. J., Computer Simulation of the Cryoprotectant Disaccharide $\alpha$, $\alpha$-Trehalose in Aqueous Solution. J. Phys. Chem. A 103 (1998), 4049-4055.

7. Cordone, L., Cottone, G., Guiffrida, S., Palazzo, G., Venturoli, G. \& Viappiani, C., Internal dynamics and protein-matrix coupling in trehalose-coated proteins. Biochemical Biophisical Acta 1749 (2005), 252-281.

8. Crowe, J. H., Hoekstra, F. A., \& Crowe, L. M., Anhydrobiosis. Annual Review of Physiology 54 (1992), 579-600. 
9. Crowe, J. H., Carpenter, J. F. \& Crowe, L. M., The role of vitrification in anhydrobiosis. Annual Review of Physiology 60 (1998), 73-103.

10. Debenedetti, P. G. \& Stillinger, F. H., Supercooled liquids and the glass transition. Nature 410 (2001), 259.

11. Doxastakis, M., Sum, A. K. \& De Pablo, J. J., Modulating membrane properties: the effect of trehalose and cholesterol on a phospholipid bilayer. Journal of Physical Chemistry 109 (2005), 2417324181.

12. Edwards, D. A. \& CohEn, D. S., An unusual moving boundary condition arising in anomalous diffusion problems. SIAM Journal on Applied Mathematics 55 (1995), 662-676. Zb10839.35148 MR1331580

13. Eroglu, A., Toner, M. \& Toth, T., Beneficial effect of microinjected trehalose on the cryosurvival of human oocytes. Fertility and Sterility 77 (2002).

14. Gruber, C. A., Moving-Boundary Problems Associated with Lyopreservation. PhD thesis, Northwestern University (2013).

15. Guo, N., Puhlev, I., Brown, D. R., Mansbridge, J. \& Levine, F., Trehalose expression confers desiccation tolerance on human cells. Nature Biotechnology 18 (2000), 168-171.

16. Lim, S. C. \& Muniandy, S. V., Self-similar Gaussian processes for modeling anomalous diffusion. Phys. Rev. E 66 (2002), 021114.

17. Lubchenko, V. \& Wolynes, P. G., Theory of structural glasses and supercooled liquids. Annu. Rev. Phys. Chem. 58 (2007), 235.

18. Lutz, E., Fractional Langevin equation. Phys. Rev. E 64 (2001).

19. Magdziarz, M., Weron, A., Burnecki, K., \& Klafter, J., Fractional brownian motion versus the continuous-time random walk: A simple test for subdiffusive dynamics. Phys. Rev. Lett. 103 (2009), 180602.

20. Mandelbrot, B. The Fractal Geometry of Nature. Freeman, New York (1983).

21. Metzler, R. \& Klafter, J., The random walk's guide to anomalous diffusion: A fractional dynamics approach. Phys. Rep. 339 (2000), 1-77. Zb10984. 82032 MR1809268

22. Metzler, R. \& Klafter, J., The restaurant at the end of the random walk: recent developments in the description of anomalous transport by fractional dynamics. J. Phys. A 37 (2004), R161-R208. Zb11075. 82018 MR2090004

23. Podlubny, I., Fractional Differential Equations. San Diego \& London, Academic Press (1999). Zbl0924.34008 MR1658022

24. Vogl, C. J., Miksis, M. J., \& DAVIS, S. H., Moving boundary problems governed by anomalous diffusion. Proc. R. Soc. A, 468: 3348-3369, 2012. MR2988264

25. Weeks, E. \& Weitz, D., Subdiffusion and the cage effect studied near the colloidal glass transition. Chem. Phys. 284 (2002), 361.

26. WiLSON, D. G., Existence and uniqueness for similarity solutions of one-dimensional multi-phase Stefan problems. Proceedings of the Royal Society A, 35 (1978), 135-147. Zbl0383. 35030 MR0473521

27. Wolfe, J. \& BRYANT, G., Freezing, drying, and/or vitrification of membrane-solute-water solutions. Cryobiology 39 (1999), 103-129. 